\title{
203 I Cardiovascular magnetic resonance assessment of myocarditis and comparison to histology and real time polymerase chain reaction: the emerging role of chlamydia
} Sophie Mavrogeni*1, Menelaos Manousakis², Konstantinos Spargias ${ }^{1}$, Pantelis Konstantoulakis ${ }^{3}$, Genovefa Kolovou ${ }^{1}$, Eftihia Demerouti ${ }^{1}$, Evangelia Papadopoulou ${ }^{1}$, Marouso Douskou ${ }^{4}$, Haralambos Moutsopoulos ${ }^{2}$ and Dennis V Cokkinos ${ }^{1}$

Address: ${ }^{1}$ Onassis Cardiac Surgery Center, Athens, Greece, ${ }^{2}$ Dept Pathophysiology, Athens University, Athens, Greece, ${ }^{3}$ Locus Medicus Lab, Athens, Greece and ${ }^{4}$ Bioiatriki MRI Unit, Athens, Greece

* Corresponding author

from I th Annual SCMR Scientific Sessions Los Angeles, CA, USA. I-3 February 2008

Published: 22 October 2008

Journal of Cardiovascular Magnetic Resonance 2008, I0(SuppI I):A300 doi:10.I I86/I532-429X-I0-SI-A300

This abstract is available from: http://jcmr-online.com/content/I0/SI/A300

(c) 2008 Mavrogeni et al; licensee BioMed Central Ltd.

\section{Introduction}

Myocarditis usually presents with spontaneous recovery. However, it can occasionally lead to sudden death in $10 \%$ or may progress to dilated cardiomyopathy in up to $9 \%$ of cases. The diagnosis is difficult to establish clinically.

\section{Purpose}

We applied cardiovascular magnetic resonance (CMR) to detect myocardial inflammation and we compared the CMR results with myocardial histology and polymerase chain reaction (PCR) findings.

\section{Methods}

Seventeen patients were prospectively studied, in whom suspicion of myocarditis had been raised. Endomyocardial biopsy was performed in all of them and submitted to histopathologic analysis. Real time PCR evaluation of myocardial speciments was also performed. CMR evaluation of myocardial inflammation was performed using T2-weighted (T2-W), T1-weighted (T1-W) before and after contrast media injection, and late enhanced images.

\section{Results}

All patients had abnormal CMR and PCR findings. Histology showed presence of myocarditis in $8 / 17$ patients
(47\%) and PCR of myocardial speciments revealed Chlamydia trachomatis in 15/17 (88\%). Coxsackie B3, B6, Parvo B19 and Herpes 1-2 were identified in 6, 5 and 4/17 patients respectively. In 10/17 patients $(58.8 \%)$ a coexistence of Chlamydia with Coxsackie B3, B6, Parvo B19 and Herpes 1-2 was revealed. Presence of oedema was documented in 6/17 patients (35.2\%) using T2W. The relative myocardial enhancement from T1-W was increased at $10 \pm 3$ (normal values $2.3 \pm 0.3$ ) in $17 / 17$ cases. Areas of late enhancement (LE), with subepicardial distribution, were located in basal posterolateral or inferior wall in 11/17 (64.7\%) cases. Left ventricular ejection fraction was reduced only in $20 \%$ of cases.

\section{Conclusion}

Chlamydia induced myocardial inflammation is a common finding in patients with clinical diagnosis of myocarditis and often coexists with different viruses. Abnormal CMR findings were in agreement with all myocardial PCR specimens and $47 \%$ of histology findings. CMR evaluation may facilitate the selection of patients for myocardial biopsy. 